\title{
Use of infectious diseases consultations by surgeons
}

\author{
Salih Hosoglu \\ Dicle University Hospital, Diyarbakir, Turkey
}

doi: 10.3396/ijic.V7i3.020.11

\begin{abstract}
This study was conducted to assess the use of infectious diseases consultation in the management of surgical site infections (SSIs) by surgeons. A cross-sectional, countrywide survey study was performed using a standardized data collection form. The form included questions about surgeons' institutions, specialties, and infectious disease (ID) consultation usage by surgeons in treatment of SSIs. Totally 326 surgeons from six different specialties at 36 hospitals in 12 cities in Turkey were included into the study. In the diagnosis of SSIs, 284 of the surgeons (87.1\%) used culture procedures, 90 (27.6\%) Gram stain, and $63(19.3 \%)$ ID consultation. On multivariate analysis, being general surgeons $(p<0.001)$, gynecologists $(p=0.017)$ and urologist $(p=0.002)$ were found significant factors for less usage of ID consultation in the treatment of SSIs. Only working at teaching hospitals was significant predictor for more usage of ID consultation in the treatment of SSIs ( $p=0.008$ ). There is a tendency among surgeons about common use culture facilities and rarely ID consultation for management of SSIs. A better collaboration among surgeons and ID specialists could be helpful for improving infection control and treatment in surgical departments.
\end{abstract}

Key words

Infectious disease consultation, surgical infection, surgeon

\section{Introduction}

Although the incidence of postoperative wound infection has been reduced remarkably during the past three decades, the diagnosis and treatment of surgical site infections (SSIs) are still important problems around the world. ${ }^{1-3}$ At the same time, there are some debates on diagnosis and treatment among surgeons and infectious disease (ID) specialists. ${ }^{4-6}$ Beside these factors, in many situations, surgeons may not use ID consultation alternative effectively. Therefore, surgeons' approach to SSIs is very important for outcome of the diseases. For many procedures, surgeons' approaches to diagnoses and treatment of post-surgical infections have significant differences and variability., $3,7,8$

\section{Correspondence}

Salih Hosoglu

Dicle University Hospital, Diyarbakir, Turkey

Email: hosoglu@hotmail.com 
In several situations, surgeons encounter various important difficulties about diagnosis and antibiotic choosing procedures in SSIs. In some cases, surgeons do not accurately and sufficiently use the ID consultation choice. However, little is known about the usage of ID consultations by Turkish surgeons. The aim of this study was to evaluate current surgeons' use of ID consultation in the management of SSIs in Turkish hospitals and to identify associated factors with diagnostic and treatment approaches.

\section{Methods}

\section{Background:}

There are 67 million people in Turkey, where the government runs most hospitals. The majority of these hospitals have ID consultants. The diagnosis of surgical site infection was made according to Healthcare Infection Control Practices Advisory Committee (HICPAC) diagnose criteria of surgical site infection. ${ }^{9}$

\section{Setting}

A cross-sectional survey was performed in 2000 and 2001, with a standardized questionnaire delivered to surgeons working at university, general public (general hospitals and social security hospitals) and private hospitals. One or two cities were included from each geographic part of Turkey. The questionnaire was delivered to surgeons working in these hospitals. The original questionnaire (Turkish version) is available from the authors on request. The questionnaire included the following items related to the standard approaching to using ID consultation practices for diagnosis and treatment of post-surgical infections by each surgeon:

(1) hospital affiliation (university, general, social security, others);

(2) hospital type (teaching or district general);

(3) how the surgeon diagnoses SSIs [radiological examination (X-Ray, ultrasound, etc.), microbiological methods (Gram staining from suspected area, culture from wound), ID consultation];

(4) and how the surgeon decides antibiotic treatment for SSIs (according to microbiological results, consulting with ID specialist, empirically).

Evaluation of the usage of ID consultation for diagnosis and treatment of SSIs

The usage of ID consultations for diagnosis and treatment of SSIs by surgeons was evaluated. The consultation with ID specialist about diagnosis of SSIs and antibiotic treatment was accepted as an indicator for a collaborative approach to SSIs.

\section{Statistical Analyses}

SPSS software (version 10.0; SPSS, Inc., Chicago, IL) was used for all data entry and analysis. In all univariate analyses, the chi-square test was used for binary variables and the Student's $t$ test was used for continuous variables. To explore the factors associated with ID specialist consultation for the management of SSIs, multivariable analysis with logistic regression was performed. Candidate variables with a $P$ value of less than 0.1 were entered using a backwards, stepwise approach. Predictor variables were retained in the final model if the $P$ value was less than 0.05. Dummy variables were used to code hospital type (university, general, social security, or other) and surgeon specialty (general surgery, gynecology, cardiac surgery, urology, orthopedics, or neurosurgery).

\section{Results}

The questionnaire was delivered to 842 surgeons and $464(55.1 \%)$ of them at 36 hospitals completed the survey. The hospitals were evenly distributed in different cities throughout Turkey (Adana, Erzurum, Elazig, Diyarbakir, Samsun, Afyon, Konya, Mersin, Izmir, Eskisehir, Kocaeli, and Denizli). One hundred and thirty eight questionnaires were excluded because of insufficient data, leaving 326 for analysis.

These 36 hospitals had 15,921 beds. This sample represented $9.1 \%$ of all 175,190 hospital-beds in Turkey during the study period. The mean number of beds per hospital was 442.3 (standard deviation, 585.0 beds; range, 30 to 1,200 beds). Fifteen (41.7\%) of the hospitals had more than 500 beds, $12(33.3 \%)$ had between 200 and 500 beds, and 9 (25.0\%) had fewer than 200 beds. The mean number of operations per month for each surgeon was 15 (standard deviation, 27 operations).

\section{The evaluation of the usage of microbiology laboratory and ID consultations for management of surgical infections}

Twenty-three of the surgeons (7.1\%) stated that they used only a physical examination for the diagnosis 
of SSIs. One hundred and nine (33.4\%) stated that they used radiological examination, 284 (87.1\%) bacteriologic culture facilities, 90 (27.6\%) Gram stain, and $63(19.3 \%)$ used ID consultation for the diagnosis of surgical infection.

In the treatment of SSIs, 284 of the surgeons (87.1\%) noted that they applied culture and antibiotic susceptibility testing to initiate and/or modify the antibiotic treatment. On the other hand, 141 of the surgeons $(43.3 \%)$ indicated that they started the antibiotic treatment in an empirical approach.

\section{Statistical Analyses}

Univariate analysis revealed that surgeons working at university hospitals, urologists and neurosurgeons apply culture facilities more frequently in the diagnostic procedures of SSIs. Surgeons working at general hospitals including Social Security Hospitals $(\mathrm{SSH})$, general surgeons and gynaecologists were using culture facilities less frequently in the diagnostic procedures. Working at teaching hospitals was a significant variable for the frequency of using culture facilities in the diagnosis of SSIs (Table I). All variables having $p \leq 0.1$ were entered into a logistic regression model. On multivariate analysis, being a general surgeon (OR, $0.369 ; \mathrm{Cl}, 0.016$ to $0.085 ; P=0.019$ ), gynaecologist $(\mathrm{OR}, 0.178 ; \mathrm{Cl}, 0.056$ to 0.564 ; $P=0.001)$ and cardiac surgeon (OR, 0.259; $\mathrm{Cl}, 0.076$ to $0.881 ; P=0.031)$ were significant factors in less frequent usage of culture procedures. Only working at a teaching hospital remained as a significant predictor for more frequent utilization of culture facilities (OR, 5.936; $\mathrm{Cl}, 2.825$ to $12.474 ; P<0.001)$.

Univariate analysis indicated that surgeons working at university hospitals, cardiac surgeons, orthopaedists and neurosurgeons were more often applying ID consultation in the treatment procedures of SSIs. Working at a SSH, being a general surgeon, gynaecologist and urologist are significant factors on less frequent usage of ID consultation in the treatment procedures. Working at teaching hospitals was a significant variable on usage frequency of ID consultation in the treatment of surgical infections (Table I). All variables having $p \leq 0.1$ were entered into a logistic regression model. On multivariate analysis, being a general surgeon $(\mathrm{OR}, 0.235 ; \mathrm{Cl}, 0.115$ to
0.483; $P<0.001)$, gynaecologist $(\mathrm{OR}, 0.084 ; \mathrm{Cl}, 0.011$ to $0.643 ; P=0.017)$ and urologist $(\mathrm{OR}, 0.040 ; \mathrm{Cl}, 0.005$ to $0.302 ; P=0.002$ ) were found as significant factors for less usage of ID consultation in the treatment of SSIs. Only working at teaching hospitals remained significant predictor for more frequent usage of ID consultation in the treatment of SSIs $(\mathrm{OR}, 2.604 ; \mathrm{Cl}$, 1.284 to $5.282 ; P=.008)$.

\section{Discussion}

The way surgeons use microbiology laboratory and ID consultation is crucial in control, diagnosis and treatment of surgical infections. Effective and early treatment may help to prevent patients from complications of post-surgical infections. ${ }^{1}$ As far as we know, this study provides the first countrywide Turkish data regarding surgeons' use of ID consultation at different types of hospitals. We believe that the studies on the diagnostic and treatment approach to SSIs can help to improve patient care and to reduce cost of diseases. Therefore, this study could be a pioneer for future survey and audit studies, as there is a need for more data to improve guidelines about control and management of surgical infections.

While the majority of surgeons (87.1\%) which participated in this study stated that they used microbiological methods, only $19.3 \%$ of surgeons indicated that they consulted ID specialists for deciding on the diagnosis of surgical infections. Moreover, about one fourth of them (25.8\%) applied ID consultation for prescribing antibiotics in the treatment of surgical infection. This result suggests that either there is not sufficient support from ID specialists for management of SSIs or no cooperation between surgeons and ID specialists. This low rate of consultation could be related to Turkish healthcare system, since surgeons had individual authority for prescribing of all antimicrobials at the time this study was conducted.

The differences in the use of culture facilities and ID consultation in the diagnosis of SSIs among hospital types could be related with hospital facilities and hospital based procedures. In Turkey, in the university hospitals, microbiology laboratory and ID consultation services are better than in the other hospitals. All university hospitals have ID specialist but some of the 


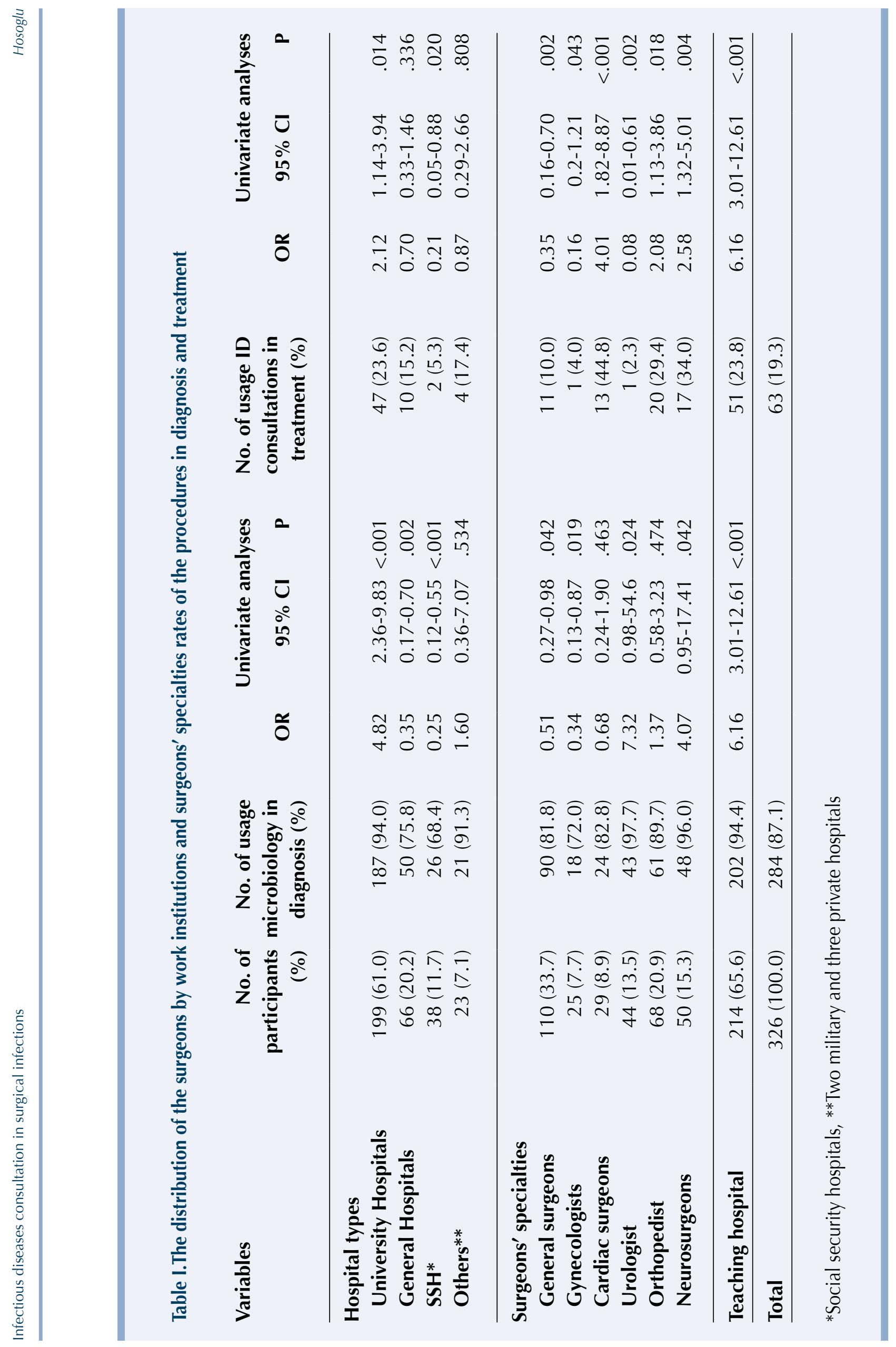


other hospitals does not. Post doctorate education of the surgeons could be better in university hospitals. The departmental differences could be related with the characteristics of infections in different departments. In the departments of urology and neurosurgery, catheter-related infections are common and they need bacteriologic examinations.

Different studies have certainly shown that ID consultation can reduce morbidity and management cost of infectious diseases. ${ }^{6,10-13}$ A special audit study on the antibiotic prescription in a Danish orthopaedic surgery department found that ID consultation significantly affect rationalization of antibiotic prescribing. ${ }^{14}$ To reduce the cost of diagnosis and treatment of surgical infection, a multidisciplinary approach could be helpful. ${ }^{12,15}$ The diagnosis of surgical infections could be easy if the infection is superficial. To achieve additional information about causative microorganisms and treatment options, microbiological tests have vital importance. As a result, a high quality surveillance program for SSIs should be supported with microbiology laboratory. To improve quality of the diagnosis and treatment of SSIs, seven days and 24 hours accessible microbiology laboratory service has a mean importance. ${ }^{8,14,16}$

Surgeons should be informed about the results of surveillance of SSIs and antibmicrobial susceptibility tests in a short time. In periodical meetings, surgeons and ID specialists (or clinical microbiologists) can discuss the results and trends of antibiotic susceptibility of the causative agents of SSIs in their hospital. ${ }^{14,17,18}$

In many countries, ID is a cognitive subspecialty that usually lacks dedicated hospital beds and does not perform unique procedures; the ID service typically provides consultations to other primary wards or services. ${ }^{5}$ For instance, in a retrospective cohort study, ID consultation resulted in $57 \%$ reduction of antibiotics costs per hospitalized day in trauma patients. ${ }^{19}$ Authors have not reached an agreement on ID specialists or surgeons' use of antibiotics in the treatment of SSIs. A few authors claimed that surgeons could make better approach than medical ID doctors about the treatment of surgical infections., ${ }^{4,20}$ Their opinions are based on the fact that ID specialists do not have enough experiences on surgical problems and they prescribe more and unnecessary antibiotics for the treatment of surgical infections. However, this topic is extremely speculative and open for debate. For example, in a survey study, ID specialists were found more conservative than the generalists to use newer antibiotics. ${ }^{21}$ In our view, if both surgeons and ID specialists make the decision collaboratively, the result could be more positive. A study from USA demonstrated that in an academic setting with a restricted formulary, the multidisciplinary antimicrobial management team had better antimicrobial prescribing than the ID fellows alone. ${ }^{22}$ For optimal antibiotic prescribing, a close monitoring of antimicrobial resistance rates of common pathogens is needed.

This study showed that collaboration among surgeons and ID specialists is not satisfactory in Turkish hospitals. For better control and management of surgical infections, surgeons could consult ID specialists in the decision making. The surgeons may apply ID consultation, at least, for the interpretation of the culture results. Close collaboration among two disciplines may improve the surgeons' approaches to control and manage surgical infections.

Conclusion: Most surgeons (87.1\%) use microbiology laboratory facilities for diagnosis of SSIs. On the other hand, a small percentage of the surgeons $(19.3 \%)$ apply ID consultation for treatment of SSIs. A good collaboration between surgeons and ID specialist can increase microbiology laboratory facilitations usage and sufficient data about nosocomial surgical infections could improve the diagnosis and treatment of SSIs. 


\section{References}

1. Wittmann DH, Schein M. Let us shorten antibiotic prophylaxis and therapy in surgery. Am J Surg 1996; 172 (supp 6A): 26S-32S.

http.dx.doi.org/10.1016/S0002-9610(96)00347-9

2. Gyssens IC. Preventing postoperative infections: Current treatment recommendations. Drugs 1999; 57: 175-185. http.dx.doi.org/10.2165/00003495-199957020-00004

3. Peterson LR, Hamilton JD, Baron EJ, et al. Role of clinical microbiology laboratories in the management and control of infectious diseases and the delivery of health care. Clin Infect Dis 2001; 32: 605-611.

http.dx.doi.org/10.1086/318725

4. Gorecki PJ, Schein M, Mehta V, Wise L. Surgeons and infectious diseases specialists: different attitudes towards antibiotic treatment and prophylaxis in common abdominal surgical infections. Surg Infect 2000; 1: 115-123. http.dx.doi.org/10.1089/109629600321155

5. Yinnon AM. Whither infectious diseases consultations? Analysis of 14,005 consultations from a 5-year period. Clin Infect Dis 2001; 33: 1661-1667.

http.dx.doi.org/10.1086/323760

6. Schlesinger Y, Paltiel O, Yinnon AM. Analysis and impact of infectious disease consultations in a general hospital. / Hosp Infect 1998; 40: 39-46. http.dx.doi.org/10.1016/S0195-6701(98)90023-8

7. Peterson LR, Noskin GA. Applying new technology for detection of multidrug-resistant pathogens in the clinical microbiology laboratory. Emerg Infect Dis 2001; 7: 306-311. http.dx.doi.org/10.3201/eid0702.010233

8. Sturm AW. Rational use of antimicrobial agents and diagnostic microbiology facilities. J Antimicrobial Chemother 1988; 22: 257-260.

http.dx.doi.org/10.1093/jac/22.2.257

9. Mangram AJ, Horan TC, Pearson ML, et al. Guideline for prevention of surgical site infection, 1999. Hospital Infection Control Practices Advisory Committee. Infect Control Hosp Epidemiol 1999; 20: 250-278. http.dx.doi.org/10.1086/501620

10. Pestotnik SL, Classen DC, Evans RS, Burke JP. Implementing antibiotic practice guidelines through computer-assisted decision support: clinical and financial outcomes. Ann Intern Med 1996; 124: 884-890.

11. Wolf S, Leitritz L, Rupp C, et al. Cost reduction after introduction of a multidisciplinary infectious disease service at a German university hospital. Infection 2000; 28: 379-383. http.dx.doi.org/10.1007/s150100070009
12. Gums JG, Yancey RW Jr, Hamilton CA, Kubilis PS. A randomized, prospective study measuring outcomes after antibiotic therapy intervention by a multidisciplinary consult team. Pharmacotherapy 1999; 19: 1369-1377. http.dx.doi.org/10.1592/phco.19.18.1369.30898

13. Fraser GL, Stogsdill P, Dickens JD Jr, et al. Antibiotic optimization. An evaluation of patient safety and economic outcomes. Arch Intern Med 1997; 157: 1689-1694.

http.dx.doi.org/10.1001/archinte.157.15.1689

14. Kolmos HJ. Interaction between the microbiology laboratory and clinician: what the microbiologist can provide. I Hosp Infect 1999; 43 Suppl: S285-291. http.dx.doi.org/10.1016/S0195-6701(99)90101-9

15. Thomas M, Govil S, Moses BV, Joseph A. Monitoring of antibiotic use in a primary and a tertiary care hospital. J Clin Epidemiol 1996; 49: 251-254. http.dx.doi.org/10.1016/0895-4356(95)00520-X

16. Sturm AW. Antibiotic advice based on an intra-operative Gram-stain during cholecystectomy. I Infect 1985; 10: 240248.

http.dx.doi.org/10.1016/S0163-4453(85)92588-5

17. Martin C, Pourriat JL. Quality of perioperative antibiotic administration by French anaesthetists. J Hosp Infect 1998; 40: $47-53$ http.dx.doi.org/10.1016/S0195-6701(98)90024-X

18. Haley RW, Culver DH, et al. Identifying patients at high risk of surgical wound infection. Am / Epidemiol 1985;121: 206215.

19. Fox BC, Imrey PB, Voights MB, Norwood S. Infectious Disease Consultation and Microbiologic Surveillance for Intensive Care Unit Trauma Patients: A Pilot Study. Clin Infect Dis 2001; 33: 1981-1989. http.dx.doi.org/10.1086/324083

20. Gorecki P, Schein M, Rucinski JC, Wise L. Antibiotic administration in patients undergoing common surgical procedures in a community teaching hospital: the chaos continues. World J Surg 1999; 23: 429-432. http.dx.doi.org/10.1007/PL00012319

21. Metlay JP, Shea JA, Asch DA. Antibiotic Prescribing Decisions of Generalists and Infectious Disease Specialists: Thresholds for Adopting New Drug Therapies. Med Decis Making 2002; 22: 498-505. http.dx.doi.org/10.1177/0272989X02238297

22. Gross R, Morgan AS, Kinky DE, et al. Impact of a HospitalBased Antimicrobial Management Program on Clinical and Economic Outcomes. Clin Infect Dis 2001; 33: 289-295. http.dx.doi.org/10.1086/321880 